Supplement of

\title{
Evaluation of EURO-CORDEX (Coordinated Regional Climate Downscaling Experiment for the Euro-Mediterranean area) historical simulations by high-quality observational datasets in southern Italy: insights on drought assessment
}

David J. Peres et al.

Correspondence to: Alfonso Senatore (alfonso.senatore@unical.it)

The copyright of individual parts of the supplement might differ from the CC BY 4.0 License. 


\section{Supplementary Material}

Table S1: Rain gauges used in the study and relative mean annual accumulated rainfall in the period 1971-2000.

\begin{tabular}{|c|c|c|c|c|c|c|}
\hline Station ID & Station Name & Region & Latitude & Longitude & Elevation & Annual mean (mm) \\
\hline C50 & Cancellara & Basilicata & 40.7340 & 15.9160 & 620 & 507 \\
\hline $\mathrm{C} 60$ & Tolve & Basilicata & 40.6981 & 16.0290 & 568 & 608 \\
\hline $\mathrm{C} 230$ & Potenza & Basilicata & 40.6370 & 15.8021 & 811 & 699 \\
\hline $\mathrm{C} 240$ & Vaglio di Lucania & Basilicata & 40.6673 & 15.9160 & 933 & 832 \\
\hline $\mathrm{C} 280$ & Calvello & Basilicata & 40.4673 & 15.8496 & 700 & 837 \\
\hline $\mathrm{C} 310$ & Cognato & Basilicata & 40.5673 & 16.1320 & 557 & 752 \\
\hline $\mathrm{C} 320$ & Calciano & Basilicata & 40.5840 & 16.1985 & 450 & 730 \\
\hline $\mathrm{C} 360$ & Pisticci & Basilicata & 40.2950 & 16.7345 & 364 & 541 \\
\hline $\mathrm{C} 410$ & San Mauro Forte & Basilicata & 40.4816 & 16.2511 & 504 & 697 \\
\hline $\mathrm{C} 470$ & Tramutola & Basilicata & 40.3253 & 15.7740 & 654 & 1078 \\
\hline $\mathrm{C} 520$ & San Martino d'Agri & Basilicata & 40.2340 & 16.0489 & 661 & 813 \\
\hline C530 & Armento & Basilicata & 40.3007 & 16.0656 & 640 & 835 \\
\hline C560 & Missanello & Basilicata & 40.2840 & 16.1652 & 566 & 875 \\
\hline C570 & Aliano & Basilicata & 40.3007 & 16.2317 & 497 & 743 \\
\hline C580 & Roccanova & Basilicata & 40.2106 & 16.1992 & 727 & 747 \\
\hline C660 & Agromonte C. C. & Basilicata & 40.0673 & 16.0489 & 500 & 1162 \\
\hline C690 & San Severino Lucano & Basilicata & 40.0173 & 16.1320 & 884 & 1417 \\
\hline $\mathrm{C} 710$ & Carbone & Basilicata & 40.1340 & 16.0822 & 685 & 1024 \\
\hline $\mathrm{C} 760$ & Senise & Basilicata & 40.1507 & 16.2815 & 330 & 668 \\
\hline $\mathrm{C} 780$ & Cersosimo & Basilicata & 40.0507 & 16.3480 & 563 & 978 \\
\hline C790 & Noepoli & Basilicata & 40.0897 & 16.3299 & 676 & 649 \\
\hline $\mathrm{C} 810$ & Valsinni & Basilicata & 40.1673 & 16.4311 & 250 & 742 \\
\hline $\mathrm{C} 820$ & Tursi & Basilicata & 40.2472 & 16.4802 & 348 & 712 \\
\hline $\mathrm{C} 830$ & Nova Siri & Basilicata & 40.1507 & 16.5307 & 300 & 621 \\
\hline $\mathrm{C} 840$ & Nova Siri Scalo & Basilicata & 40.1340 & 16.6304 & 2 & 539 \\
\hline $\mathrm{C} 850$ & Nocara & Calabria & 40.0856 & 16.4817 & 830 & 621 \\
\hline C860 & Montegiordano Scalo & Calabria & 40.0406 & 16.5981 & 7 & 467 \\
\hline $\mathrm{C} 880$ & Castroregio & Calabria & 39.9937 & 16.4787 & 820 & 631 \\
\hline C920 & Trebisacce & Calabria & 39.8656 & 16.5306 & 10 & 532 \\
\hline C930 & Villapiana Scalo & Calabria & 39.7973 & 16.4819 & 5 & 479 \\
\hline C940 & Francavilla Marittima & Calabria & 39.8184 & 16.3851 & 272 & 618 \\
\hline C950 & San Lorenzo Bellizzi & Calabria & 39.8887 & 16.3290 & 851 & 928 \\
\hline C970 & Cassano allo Ionio & Calabria & 39.7831 & 16.3189 & 251 & 686 \\
\hline C980 & Piane Crati & Calabria & 39.2326 & 16.3229 & 583 & 1066 \\
\hline C990 & Trenta & Calabria & 39.2801 & 16.3245 & 534 & 757 \\
\hline $\mathrm{C} 1000$ & Domanico & Calabria & 39.2164 & 16.2075 & 736 & 1473 \\
\hline
\end{tabular}




\begin{tabular}{|c|c|}
\hline C1010 & Cosenza \\
\hline C1020 & Cerisano \\
\hline C1030 & San Pietro in Guarano \\
\hline C1040 & Rende \\
\hline $\mathrm{C} 1050$ & Rose \\
\hline $\mathrm{C} 1070$ & Laghitello C.C. \\
\hline C1080 & San Martino di Finita \\
\hline $\mathrm{C} 1100$ & Cecita \\
\hline $\mathrm{C} 1110$ & Pinutello C.C. \\
\hline $\mathrm{C} 1120$ & Acri \\
\hline $\mathrm{C} 1130$ & Torano Scalo \\
\hline $\mathrm{C} 1150$ & Santa Sofia d'Epiro \\
\hline $\mathrm{C} 1170$ & Morano Calabro \\
\hline $\mathrm{C} 1180$ & Castrovillari \\
\hline C1190 & Firmo \\
\hline $\mathrm{C} 1220$ & Roggiano Gravina \\
\hline $\mathrm{C} 1230$ & San Sosti \\
\hline $\mathrm{C} 1260$ & San Marco Argentano \\
\hline C1300 & San Giorgio Albanese \\
\hline $\mathrm{C} 1310$ & Schiavonea \\
\hline $\mathrm{C} 1320$ & San Giacomo d'Acri \\
\hline $\mathrm{C} 1330$ & Rossano \\
\hline $\mathrm{C} 1360$ & Longobucco \\
\hline $\mathrm{C} 1370$ & Bocchigliero \\
\hline $\mathrm{C} 1380$ & Cropalati \\
\hline C1390 & Crosia \\
\hline $\mathrm{C} 1410$ & Cariati Marina \\
\hline C1430 & Scala Coeli \\
\hline C1440 & Crucoli \\
\hline C1460 & Ciro' Marina \\
\hline $\mathrm{C} 1470$ & San Giovanni in Fiore \\
\hline $\mathrm{C} 1500$ & Nocelle - Arvo \\
\hline $\mathrm{C} 1510$ & Sculca \\
\hline $\mathrm{C} 1540$ & Berberano C.C. \\
\hline $\mathrm{C} 1550$ & Trepido' \\
\hline $\mathrm{C} 1560$ & Casa Pasquale \\
\hline $\mathrm{C} 1590$ & Belvedere Spinello \\
\hline C1620 & Verzino \\
\hline $\mathrm{C} 1650$ & Strongoli \\
\hline C1680 & Crotone \\
\hline
\end{tabular}

\begin{tabular}{|c|c|c|c|c|}
\hline Calabria & 39.2869 & 16.2653 & 242 & 884 \\
\hline Calabria & 39.2811 & 16.1743 & 620 & 1492 \\
\hline Calabria & 39.3456 & 16.3135 & 660 & 943 \\
\hline Calabria & 39.3312 & 16.1804 & 482 & 1101 \\
\hline Calabria & 39.3984 & 16.2876 & 433 & 986 \\
\hline Calabria & 39.4381 & 16.1012 & 870 & 1625 \\
\hline Calabria & 39.4898 & 16.1081 & 470 & 1684 \\
\hline Calabria & 39.4000 & 16.5378 & 1180 & 943 \\
\hline Calabria & 39.4515 & 16.4743 & 1005 & 1138 \\
\hline Calabria & 39.4826 & 16.3935 & 790 & 965 \\
\hline Calabria & 39.4938 & 16.2098 & 97 & 796 \\
\hline Calabria & 39.5476 & 16.3201 & 550 & 894 \\
\hline Calabria & 39.8401 & 16.1380 & 722 & 1183 \\
\hline Calabria & 39.7655 & 16.2542 & 353 & 757 \\
\hline Calabria & 39.7220 & 16.1742 & 1369 & 743 \\
\hline Calabria & 39.6179 & 16.1504 & 264 & 509 \\
\hline Calabria & 39.6644 & 16.0279 & 404 & 1517 \\
\hline Calabria & 39.5594 & 16.1108 & 430 & 1216 \\
\hline Calabria & 39.5834 & 16.4529 & 430 & 897 \\
\hline Calabria & 39.6534 & 16.5392 & 3 & 415 \\
\hline Calabria & 39.5242 & 16.4651 & 724 & 983 \\
\hline Calabria & 39.5745 & 16.6343 & 300 & 894 \\
\hline Calabria & 39.4461 & 16.6103 & 770 & 1144 \\
\hline Calabria & 39.4173 & 16.7529 & 870 & 1134 \\
\hline Calabria & 39.5157 & 16.7245 & 367 & 902 \\
\hline Calabria & 39.5667 & 16.7729 & 279 & 687 \\
\hline Calabria & 39.5051 & 16.9402 & 10 & 814 \\
\hline Calabria & 39.4454 & 16.8898 & 330 & 1126 \\
\hline Calabria & 39.4245 & 17.0038 & 367 & 902 \\
\hline Calabria & 39.4079 & 17.1093 & 6 & 689 \\
\hline Calabria & 39.2633 & 16.7157 & 1050 & 1131 \\
\hline Calabria & 39.2432 & 16.5449 & 1315 & 1138 \\
\hline Calabria & 39.3220 & 16.5023 & 1358 & 1256 \\
\hline Calabria & 39.1817 & 16.5748 & 1280 & 1117 \\
\hline Calabria & 39.1887 & 16.6979 & 1295 & 1117 \\
\hline Calabria & 39.1812 & 16.7190 & 1246 & 1350 \\
\hline Calabria & 39.2071 & 16.8878 & 330 & 829 \\
\hline Calabria & 39.3131 & 16.8620 & 550 & 915 \\
\hline Calabria & 39.2645 & 17.0470 & 342 & 667 \\
\hline Calabria & 39.0870 & 17.1310 & 5 & 656 \\
\hline
\end{tabular}




\begin{tabular}{|c|c|}
\hline C1690 & Capo Colonne \\
\hline $\mathrm{C} 1720$ & Steccato \\
\hline C1730 & Petilia Policastro \\
\hline $\mathrm{C} 1760$ & Botricello \\
\hline $\mathrm{C} 1780$ & Cropani \\
\hline C1800 & Sellia Marina \\
\hline C1830 & Albi \\
\hline C1840 & Sant'Elia \\
\hline $\mathrm{C} 1850$ & Catanzaro \\
\hline C1860 & Catanzaro Lido \\
\hline C1880 & Fiorenza \\
\hline C1890 & Umbri \\
\hline C1920 & Borgia \\
\hline C1940 & Palermiti \\
\hline C1950 & Stalettì \\
\hline C1960 & Chiaravalle Centrale \\
\hline C1980 & Serra San Bruno \\
\hline C1990 & Simbario \\
\hline C2010 & San Sostene \\
\hline C2040 & Monasterace - Punta Stilo \\
\hline C2070 & Riace \\
\hline C2080 & Placanica \\
\hline C2120 & Caulonia \\
\hline C2130 & Roccella Ionica \\
\hline $\mathrm{C} 2150$ & Fabrizia - Cassari \\
\hline C2160 & Gioiosa Ionica \\
\hline C2170 & Siderno Marina \\
\hline $\mathrm{C} 2180$ & Antonimina - Canolo Nuovo \\
\hline $\mathrm{C} 2210$ & Ardore Superiore \\
\hline C2230 & Plati' \\
\hline $\mathrm{C} 2260$ & San Luca \\
\hline $\mathrm{C} 2270$ & Sant'Agata del Bianco \\
\hline C2290 & Staiti \\
\hline C2310 & Capo Spartivento \\
\hline $\mathrm{C} 2320$ & Bova Superiore \\
\hline $\mathrm{C} 2330$ & Bova Marina \\
\hline $\mathrm{C} 2350$ & San Carlo \\
\hline $\mathrm{C} 2370$ & Melito di Porto Salvo \\
\hline C2380 & Montebello Ionico \\
\hline C2390 & Capo dell'Armi \\
\hline
\end{tabular}

\begin{tabular}{|c|c|c|c|c|}
\hline Calabria & 39.0287 & 17.2043 & 24 & 536 \\
\hline Calabria & 38.9448 & 16.9229 & 15 & 600 \\
\hline Calabria & 39.1108 & 16.7877 & 434 & 977 \\
\hline Calabria & 38.9415 & 16.8586 & 18 & 449 \\
\hline Calabria & 38.9710 & 16.7800 & 347 & 905 \\
\hline Calabria & 38.9060 & 16.7436 & 30 & 684 \\
\hline Calabria & 39.0264 & 16.5982 & 710 & 1193 \\
\hline Calabria & 38.9645 & 16.5862 & 650 & 1158 \\
\hline Calabria & 38.9108 & 16.5859 & 334 & 971 \\
\hline Calabria & 38.8206 & 16.6129 & 6 & 661 \\
\hline Calabria & 39.0923 & 16.4934 & 1126 & 1275 \\
\hline Calabria & 39.0259 & 16.5134 & 885 & 1078 \\
\hline Calabria & 38.8270 & 16.5076 & 332 & 958 \\
\hline Calabria & 38.7492 & 16.4528 & 480 & 1229 \\
\hline Calabria & 38.7654 & 16.5388 & 390 & 873 \\
\hline Calabria & 38.6713 & 16.4088 & 714 & 1369 \\
\hline Calabria & 38.5652 & 16.3197 & 790 & 1652 \\
\hline Calabria & 38.6112 & 16.3268 & 760 & 1429 \\
\hline Calabria & 38.6375 & 16.4868 & 475 & 1442 \\
\hline Calabria & 38.4537 & 16.5522 & 70 & 669 \\
\hline Calabria & 38.4179 & 16.4781 & 304 & 896 \\
\hline Calabria & 38.4145 & 16.4704 & 250 & 916 \\
\hline Calabria & 38.3832 & 16.4114 & 275 & 921 \\
\hline Calabria & 38.3216 & 16.3998 & 5 & 754 \\
\hline Calabria & 38.4421 & 16.2933 & 970 & 1620 \\
\hline Calabria & 38.3011 & 16.3298 & 125 & 893 \\
\hline Calabria & 38.2741 & 16.2985 & 7 & 672 \\
\hline Calabria & 38.3131 & 16.1272 & 880 & 1736 \\
\hline Calabria & 38.2059 & 16.1550 & 250 & 895 \\
\hline Calabria & 38.2222 & 16.0444 & 300 & 1643 \\
\hline Calabria & 38.1484 & 16.0672 & 250 & 1002 \\
\hline Calabria & 38.1007 & 16.0853 & 380 & 977 \\
\hline Calabria & 37.9998 & 16.0354 & 550 & 1004 \\
\hline Calabria & 37.9273 & 16.0561 & 48 & 631 \\
\hline Calabria & 37.9961 & 15.9328 & 905 & 778 \\
\hline Calabria & 37.9313 & 15.9215 & 8 & 461 \\
\hline Calabria & 37.9592 & 15.8903 & 76 & 546 \\
\hline Calabria & 37.9200 & 15.7714 & 7 & 528 \\
\hline Calabria & 37.9839 & 15.7584 & 470 & 823 \\
\hline Calabria & 37.9648 & 15.6842 & 117 & 504 \\
\hline
\end{tabular}




\begin{tabular}{|c|c|c|c|c|c|c|}
\hline $\mathrm{C} 2400$ & Motta San Giovanni & Calabria & 38.0026 & 15.6945 & 480 & 553 \\
\hline $\mathrm{C} 2420$ & Armo & Calabria & 38.0703 & 15.7139 & 349 & 1052 \\
\hline $\mathrm{C} 2480$ & Gallico Marina & Calabria & 38.1656 & 15.6470 & 10 & 803 \\
\hline $\mathrm{C} 2510$ & Scilla & Calabria & 38.2516 & 15.7197 & 73 & 735 \\
\hline $\mathrm{C} 2560$ & Sinopoli & Calabria & 38.2599 & 15.8774 & 502 & 1265 \\
\hline $\mathrm{C} 2570$ & Oppido Mamertina - Castellace & Calabria & 38.3124 & 15.9401 & 189 & 1512 \\
\hline $\mathrm{C} 2580$ & Molochio & Calabria & 38.3088 & 16.0313 & 310 & 1038 \\
\hline $\mathrm{C} 2600$ & Cittanova & Calabria & 38.3522 & 16.0775 & 407 & 1396 \\
\hline $\mathrm{C} 2610$ & Rizziconi & Calabria & 38.4077 & 15.9716 & 114 & 1171 \\
\hline $\mathrm{C} 2640$ & Filogaso & Calabria & 38.6803 & 16.2306 & 286 & 933 \\
\hline $\mathrm{C} 2670$ & Arena & Calabria & 38.5621 & 16.2169 & 450 & 1040 \\
\hline C2680 & San Pier Fedele & Calabria & 38.5240 & 16.1428 & 325 & 999 \\
\hline C2690 & Feroleto della Chiesa & Calabria & 38.4681 & 16.0684 & 160 & 991 \\
\hline $\mathrm{C} 2720$ & Polistena & Calabria & 38.4065 & 16.0745 & 239 & 1036 \\
\hline $\mathrm{C} 2730$ & Mileto & Calabria & 38.6272 & 16.0669 & 368 & 830 \\
\hline $\mathrm{C} 2740$ & Rosarno & Calabria & 38.5005 & 15.9880 & 61 & 898 \\
\hline $\mathrm{C} 2750$ & Calimera & Calabria & 38.5567 & 16.0187 & 180 & 879 \\
\hline $\mathrm{C} 2760$ & Joppolo & Calabria & 38.5920 & 15.9052 & 185 & 823 \\
\hline $\mathrm{C} 2770$ & Tropea & Calabria & 38.6753 & 15.8944 & 51 & 742 \\
\hline $\mathrm{C} 2780$ & Zungri & Calabria & 38.6535 & 15.9829 & 578 & 861 \\
\hline $\mathrm{C} 2790$ & Briatico & Calabria & 38.7270 & 16.0365 & 25 & 759 \\
\hline $\mathrm{C} 2830$ & Filadelfia & Calabria & 38.7869 & 16.2930 & 550 & 1183 \\
\hline $\mathrm{C} 2840$ & Torre Mezzapraia & Calabria & 38.8209 & 16.2204 & 20 & 848 \\
\hline $\mathrm{C} 2850$ & Curinga Scalo & Calabria & 38.8401 & 16.2612 & 25 & 805 \\
\hline $\mathrm{C} 2860$ & San Tommaso & Calabria & 39.0862 & 16.3768 & 820 & 1374 \\
\hline $\mathrm{C} 2870$ & Decollatura & Calabria & 39.0464 & 16.3582 & 780 & 1249 \\
\hline C2890 & Tiriolo & Calabria & 38.9395 & 16.5099 & 690 & 1172 \\
\hline C2900 & Marcellinara & Calabria & 38.9287 & 16.4943 & 330 & 1048 \\
\hline $\mathrm{C} 2960$ & Sant'Eufemia Lamezia & Calabria & 38.9215 & 16.2523 & 25 & 843 \\
\hline C2980 & Savuto C.C. & Calabria & 39.1626 & 16.5034 & 1205 & 921 \\
\hline C 3000 & Rogliano & Calabria & 39.1835 & 16.3257 & 650 & 1113 \\
\hline C3020 & Nocera Terinese & Calabria & 39.0378 & 16.1663 & 250 & 1281 \\
\hline C3040 & Amantea & Calabria & 39.1492 & 16.0862 & 54 & 931 \\
\hline C 3050 & Fiumefreddo Bruzio & Calabria & 39.2351 & 16.0710 & 220 & 965 \\
\hline C3060 & Paola & Calabria & 39.3673 & 16.0420 & 160 & 971 \\
\hline C3080 & Guardia Piemontese & Calabria & 39.4667 & 15.9998 & 515 & 1210 \\
\hline C3090 & Cetraro Superiore & Calabria & 39.5351 & 15.9640 & 416 & 968 \\
\hline $\mathrm{C} 3150$ & Laino Borgo & Calabria & 39.9544 & 15.9760 & 250 & 1293 \\
\hline C 3160 & Campotenese & Calabria & 39.8726 & 16.0684 & 965 & 1493 \\
\hline C3190 & Orsomarso & Calabria & 39.8001 & 15.9095 & 120 & 976 \\
\hline
\end{tabular}




\begin{tabular}{|c|c|}
\hline C3200 & Scalea \\
\hline C3210 & Lagonegro \\
\hline C3230 & Trecchina \\
\hline C3240 & Lauria Inferiore \\
\hline $\mathrm{C} 3250$ & Aieta \\
\hline $\mathrm{C} 3260$ & Maratea \\
\hline $\mathrm{S} 10$ & Calvaruso \\
\hline S20 & San Saba \\
\hline S40 & Monforte S. Giorgio \\
\hline $\mathrm{S} 50$ & Santa Lucia del Mela \\
\hline S70 & Castroreale \\
\hline S80 & Barcellona Pozzo di Gotto \\
\hline $\mathrm{S} 100$ & Rodi Milici \\
\hline $\mathrm{S} 120$ & Casale Tripi \\
\hline $\mathrm{S} 130$ & Montalbano Elicona \\
\hline $\mathrm{S} 140$ & Tindari \\
\hline $\mathrm{S} 150$ & Raccuia \\
\hline S160 & Ficarra \\
\hline S170 & Tortorici \\
\hline $\mathrm{S} 220$ & Militello Rosmarino \\
\hline S270 & Alcara Li Fusi \\
\hline S310 & San Fratello \\
\hline S320 & Caronia \\
\hline S330 & Mistretta \\
\hline S345 & Santo Stefano di Camastra \\
\hline $\mathrm{S} 350$ & Castel di Lucio \\
\hline S360 & Tusa \\
\hline S370 & San Mauro Castelverde \\
\hline S380 & Geraci Siculo \\
\hline S430 & Castelbuono \\
\hline S470 & Cefalù \\
\hline $\mathrm{S} 480$ & Collesano \\
\hline S520 & Scillato \\
\hline S530 & Caltavuturo \\
\hline S580 & Cerda \\
\hline S596 & Termini Imerese \\
\hline S600 & Vicari \\
\hline S610 & Campofelice d'Italia \\
\hline S630 & Mezzojuso \\
\hline S650 & Caccamo \\
\hline
\end{tabular}

\begin{tabular}{|c|c|c|c|c|}
\hline Calabria & 39.8111 & 15.7923 & 10 & 892 \\
\hline Basilicata & 40.1342 & 15.7620 & 666 & 1674 \\
\hline Basilicata & 40.0173 & 15.7831 & 500 & 1677 \\
\hline Basilicata & 40.0507 & 15.8330 & 630 & 1496 \\
\hline Calabria & 39.9292 & 15.8237 & 524 & 1298 \\
\hline Basilicata & 39.9840 & 15.7167 & 300 & 1217 \\
\hline Sicily & 38.2242 & 15.4551 & 135 & 1000 \\
\hline Sicily & 38.2828 & 15.4978 & 24 & 761 \\
\hline Sicily & 38.1589 & 15.3842 & 293 & 888 \\
\hline Sicily & 38.1421 & 15.2844 & 280 & 817 \\
\hline Sicily & 38.0999 & 15.2118 & 399 & 829 \\
\hline Sicily & 38.1505 & 15.2193 & 49 & 745 \\
\hline Sicily & 38.0839 & 15.1692 & 160 & 748 \\
\hline Sicily & 38.0507 & 15.1007 & 430 & 692 \\
\hline Sicily & 38.0267 & 15.0098 & 907 & 974 \\
\hline Sicily & 38.1444 & 15.0449 & 280 & 716 \\
\hline Sicily & 38.0535 & 14.9667 & 650 & 807 \\
\hline Sicily & 38.0576 & 14.9099 & 541 & 877 \\
\hline Sicily & 38.1100 & 14.8303 & 475 & 1039 \\
\hline Sicily & 38.0318 & 14.8253 & 459 & 940 \\
\hline Sicily & 38.0453 & 14.6748 & 400 & 952 \\
\hline Sicily & 38.0249 & 14.6990 & 690 & 858 \\
\hline Sicily & 38.0214 & 14.4429 & 302 & 620 \\
\hline Sicily & 37.9271 & 14.3625 & 910 & 910 \\
\hline Sicily & 37.8673 & 14.3979 & 135 & 602 \\
\hline Sicily & 37.8881 & 14.3130 & 772 & 837 \\
\hline Sicily & 37.9862 & 14.2370 & 535 & 819 \\
\hline Sicily & 37.9160 & 14.1920 & 1109 & 810 \\
\hline Sicily & 37.8639 & 14.1554 & 1070 & 858 \\
\hline Sicily & 37.9315 & 14.0898 & 428 & 819 \\
\hline Sicily & 38.0356 & 14.0170 & 30 & 637 \\
\hline Sicily & 37.9198 & 13.9383 & 481 & 812 \\
\hline Sicily & 37.8639 & 13.9150 & 376 & 700 \\
\hline Sicily & 37.8209 & 13.8921 & 635 & 686 \\
\hline Sicily & 37.9033 & 13.8170 & 274 & 622 \\
\hline Sicily & 37.9864 & 13.6895 & 75 & 513 \\
\hline Sicily & 37.8293 & 13.5705 & 650 & 512 \\
\hline Sicily & 37.8282 & 13.4876 & 730 & 683 \\
\hline Sicily & 37.8669 & 13.4668 & 517 & 749 \\
\hline Sicily & 37.9342 & 13.6652 & 521 & 631 \\
\hline
\end{tabular}




\begin{tabular}{|c|c|c|c|c|c|c|}
\hline S660 & Ciminna & Sicily & 37.8957 & 13.5598 & 525 & 608 \\
\hline S690 & Capo Zafferano & Sicily & 38.1138 & 13.5382 & 22 & 410 \\
\hline$S 720$ & Ficuzza & Sicily & 37.8868 & 13.3793 & 681 & 753 \\
\hline S730 & Marineo & Sicily & 37.9542 & 13.4153 & 540 & 753 \\
\hline S760 & San Ciro & Sicily & 38.0888 & 13.3874 & 100 & 554 \\
\hline S780 & Pioppo & Sicily & 38.0506 & 13.2331 & 495 & 1066 \\
\hline S790 & Altofonte & Sicily & 38.0443 & 13.2953 & 354 & 856 \\
\hline S827 & San Martino delle Scale & Sicily & 38.0911 & 13.2539 & 570 & 900 \\
\hline S850 & Partinico & Sicily & 38.0469 & 13.1177 & 189 & 670 \\
\hline S860 & Cinisi & Sicily & 38.1572 & 13.1074 & 120 & 660 \\
\hline S875 & Palermo (Osservatorio) & Sicily & 38.1062 & 13.3346 & 37 & 480 \\
\hline S920 & Palermo Sez. Idr. & Sicily & 38.1210 & 13.3579 & 19 & 584 \\
\hline S950 & San Giuseppe Jato & Sicily & 37.9748 & 13.1869 & 462 & 722 \\
\hline S975 & Alcamo & Sicily & 37.9798 & 12.9182 & 256 & 704 \\
\hline S980 & Calatafimi & Sicily & 37.9167 & 12.8667 & 350 & 682 \\
\hline S1010 & Lentina & Sicily & 38.0539 & 12.6756 & 90 & 571 \\
\hline S1030 & Trapani & Sicily & 38.0164 & 12.5072 & 2 & 454 \\
\hline S1050 & San Vito lo Capo & Sicily & 38.1901 & 12.7332 & 3 & 486 \\
\hline S1054 & Specchia & Sicily & 37.9631 & 12.6795 & 140 & 501 \\
\hline S1060 & Fastaia & Sicily & 37.9338 & 12.7338 & 218 & 552 \\
\hline S1110 & Ciavolo & Sicily & 37.7776 & 12.5491 & 120 & 522 \\
\hline S1120 & Marsala & Sicily & 37.7990 & 12.4387 & 12 & 456 \\
\hline S1130 & Petrosino (ex Milo) & Sicily & 37.7158 & 12.4919 & 2 & 432 \\
\hline S1144 & Mazara del Vallo & Sicily & 37.6586 & 12.5994 & 8 & 480 \\
\hline S1150 & Salemi & Sicily & 37.8194 & 12.8014 & 340 & 580 \\
\hline S1180 & Partanna & Sicily & 37.7311 & 12.8922 & 407 & 630 \\
\hline S1190 & Castelvetrano & Sicily & 37.6928 & 12.8006 & 190 & 490 \\
\hline S1209 & Piana dei Greci (Maganoce) & Sicily & 38.0016 & 13.2696 & 616 & 823 \\
\hline S1230 & Santa Cristina Gela & Sicily & 37.9872 & 13.3270 & 656 & 1040 \\
\hline S1270 & Corleone & Sicily & 37.8162 & 13.3019 & 588 & 644 \\
\hline S1300 & Roccamena & Sicily & 37.8381 & 13.1544 & 480 & 531 \\
\hline S1310 & Gibellina & Sicily & 37.7900 & 12.9727 & 410 & 608 \\
\hline S1340 & Menfi & Sicily & 37.6027 & 12.9632 & 119 & 502 \\
\hline S1360 & Sambuca di Sicilia & Sicily & 37.6506 & 13.1114 & 296 & 625 \\
\hline S1380 & Sciacca & Sicily & 37.5130 & 13.0821 & 56 & 518 \\
\hline S1405 & Caltabellotta & Sicily & 37.5778 & 13.2159 & 949 & 646 \\
\hline S1410 & Prizzi & Sicily & 37.7233 & 13.4314 & 1100 & 715 \\
\hline S1420 & Piano del Leone & Sicily & 37.6735 & 13.4668 & 831 & 752 \\
\hline S1430 & Palazzo Adriano & Sicily & 37.6856 & 13.3801 & 679 & 729 \\
\hline S1440 & Chiusa Sclafani & Sicily & 37.6783 & 13.2707 & 614 & 696 \\
\hline
\end{tabular}




\begin{tabular}{|c|c|}
\hline $\mathrm{S} 1450$ & Burgio \\
\hline S1460 & Ribera \\
\hline S1480 & Bivona \\
\hline S1490 & Lercara Friddi \\
\hline S1540 & Castronovo di Sicilia \\
\hline S1550 & Casteltermini \\
\hline S1560 & San Cataldo \\
\hline S1600 & Santa Caterina Villermosa \\
\hline S1610 & Valledolmo \\
\hline S1630 & Marianopoli \\
\hline S1660 & Vallelunga Pratameno \\
\hline S1680 & Mussomeli \\
\hline S1700 & Racalmuto \\
\hline S1710 & Bompensiere \\
\hline S1720 & San Biagio Platani \\
\hline $\mathrm{S} 1750$ & Cianciana \\
\hline S1760 & Cattolica Eraclea \\
\hline S1780 & Raffadali \\
\hline S1810 & Agrigento (Ispettorato) \\
\hline S1830 & Castrofilippo \\
\hline S1840 & Canicattì \\
\hline S1850 & Palma di Montechiaro \\
\hline S1860 & Petralia Sottana \\
\hline S1900 & Alimena \\
\hline S1920 & Resuttano \\
\hline S1960 & Enna \\
\hline S1980 & Villapriolo \\
\hline S1990 & Villarosa \\
\hline S2000 & Granci (Fattoria) \\
\hline S2020 & Caltanissetta \\
\hline S2040 & Pietraperzia \\
\hline S2100 & Sommatino \\
\hline S2150 & Ravanusa \\
\hline S2200 & Licata \\
\hline S2220 & Butera \\
\hline S2240 & Gela \\
\hline S2248 & Mazzarino \\
\hline S2250 & Piazza Armerina \\
\hline S2290 & Monterosso Almo \\
\hline S2300 & Chiaramonte Gulfi \\
\hline
\end{tabular}

\begin{tabular}{|c|c|c|c|c|}
\hline Sicily & 37.6031 & 13.2902 & 378 & 666 \\
\hline Sicily & 37.5003 & 13.2713 & 230 & 540 \\
\hline Sicily & 37.6209 & 13.4396 & 521 & 794 \\
\hline Sicily & 37.7490 & 13.6005 & 658 & 549 \\
\hline Sicily & 37.6814 & 13.6054 & 661 & 708 \\
\hline Sicily & 37.5431 & 13.6425 & 577 & 616 \\
\hline Sicily & 37.4865 & 13.9793 & 643 & 577 \\
\hline Sicily & 37.5895 & 14.0299 & 606 & 544 \\
\hline Sicily & 37.7485 & 13.8275 & 750 & 606 \\
\hline Sicily & 37.5985 & 13.9150 & 700 & 403 \\
\hline Sicily & 37.6853 & 13.8319 & 460 & 600 \\
\hline Sicily & 37.5811 & 13.7541 & 761 & 569 \\
\hline Sicily & 37.4100 & 13.7341 & 450 & 585 \\
\hline Sicily & 37.4745 & 13.7832 & 260 & 633 \\
\hline Sicily & 37.5112 & 13.5245 & 408 & 610 \\
\hline Sicily & 37.5223 & 13.4334 & 439 & 587 \\
\hline Sicily & 37.4421 & 13.3971 & 150 & 496 \\
\hline Sicily & 37.4059 & 13.5327 & 297 & 658 \\
\hline Sicily & 37.3130 & 13.5873 & 175 & 481 \\
\hline Sicily & 37.3511 & 13.7500 & 475 & 583 \\
\hline Sicily & 37.3595 & 13.8504 & 470 & 578 \\
\hline Sicily & 37.1942 & 13.7645 & 135 & 468 \\
\hline Sicily & 37.8092 & 14.0924 & 930 & 794 \\
\hline Sicily & 37.6897 & 14.1146 & 775 & 538 \\
\hline Sicily & 37.6813 & 14.0291 & 555 & 588 \\
\hline Sicily & 37.5696 & 14.2731 & 950 & 656 \\
\hline Sicily & 37.6325 & 14.1882 & 565 & 564 \\
\hline Sicily & 37.5873 & 14.1755 & 518 & 509 \\
\hline Sicily & 37.4923 & 14.2398 & 572 & 544 \\
\hline Sicily & 37.4978 & 14.0577 & 375 & 510 \\
\hline Sicily & 37.4222 & 14.1386 & 467 & 483 \\
\hline Sicily & 37.3391 & 13.9959 & 349 & 474 \\
\hline Sicily & 37.2693 & 13.9746 & 306 & 492 \\
\hline Sicily & 37.1025 & 13.9343 & 70 & 433 \\
\hline Sicily & 37.1895 & 14.1849 & 390 & 477 \\
\hline Sicily & 37.0652 & 14.2520 & 30 & 373 \\
\hline Sicily & 37.3044 & 14.2190 & 547 & 524 \\
\hline Sicily & 37.3673 & 14.3346 & 721 & 643 \\
\hline Sicily & 37.0890 & 14.7631 & 667 & 673 \\
\hline Sicily & 37.0302 & 14.7037 & 672 & 756 \\
\hline
\end{tabular}




\begin{tabular}{|c|c|}
\hline S2345 & Acate \\
\hline $\mathrm{S} 2350$ & Vittoria \\
\hline S2370 & Ragusa \\
\hline S2380 & Modica \\
\hline S2390 & Scicli \\
\hline S2410 & Ispica \\
\hline S2420 & Cozzo Spadaro \\
\hline S2430 & Rosolini \\
\hline S2450 & Noto \\
\hline S2468 & Canicattini Bagni \\
\hline S2470 & Palazzolo Acreide \\
\hline S2490 & Sortino \\
\hline S2510 & Floridia \\
\hline S2540 & Siracusa \\
\hline S2550 & Augusta \\
\hline S2570 & Francofonte \\
\hline S2580 & Lentini (Città) \\
\hline S2690 & Cesarò \\
\hline S2750 & Troina \\
\hline S2780 & Maletto \\
\hline S2790 & Bronte \\
\hline S2800 & Nicosia \\
\hline S2810 & Capizzi \\
\hline S2830 & Cerami \\
\hline S2840 & Gagliano Castelferrato \\
\hline S2870 & Ragalna \\
\hline S2880 & Centuripe \\
\hline S2900 & Adrano \\
\hline S2910 & Paternò \\
\hline S2930 & Motta San Anastasia \\
\hline S2935 & Simeto (Stazione) \\
\hline S2940 & Leonforte \\
\hline S2960 & Valguarnera Caropepe \\
\hline S2980 & Catenanuova \\
\hline S2990 & Castel di Judica \\
\hline S3020 & Aidone \\
\hline S3030 & Mirabella Imbaccari \\
\hline S3050 & Caltagirone \\
\hline S3060 & Mineo \\
\hline S3070 & Ramacca \\
\hline
\end{tabular}

\begin{tabular}{|c|c|c|c|c|}
\hline Sicily & 36.9984 & 14.4266 & 199 & 429 \\
\hline Sicily & 36.9531 & 14.5266 & 168 & 456 \\
\hline Sicily & 36.9242 & 14.7244 & 515 & 617 \\
\hline Sicily & 36.8605 & 14.7625 & 370 & 600 \\
\hline Sicily & 36.7946 & 14.7068 & 130 & 484 \\
\hline Sicily & 36.7864 & 14.9164 & 127 & 468 \\
\hline Sicily & 36.6884 & 15.1321 & 50 & 481 \\
\hline Sicily & 36.8221 & 14.9522 & 137 & 611 \\
\hline Sicily & 36.8927 & 15.0713 & 76 & 654 \\
\hline Sicily & 37.0369 & 15.0608 & 362 & 835 \\
\hline Sicily & 37.0676 & 14.9074 & 695 & 642 \\
\hline Sicily & 37.1620 & 15.0278 & 435 & 798 \\
\hline Sicily & 37.0831 & 15.1533 & 111 & 652 \\
\hline Sicily & 37.0626 & 15.2928 & 23 & 524 \\
\hline Sicily & 37.2307 & 15.2245 & 11 & 536 \\
\hline Sicily & 37.2276 & 14.8811 & 281 & 676 \\
\hline Sicily & 37.2926 & 14.9989 & 43 & 582 \\
\hline Sicily & 37.8469 & 14.7099 & 1100 & 710 \\
\hline Sicily & 37.7839 & 14.5982 & 1074 & 604 \\
\hline Sicily & 37.8302 & 14.8659 & 950 & 641 \\
\hline Sicily & 37.7869 & 14.8335 & 780 & 582 \\
\hline Sicily & 37.7486 & 14.3973 & 650 & 719 \\
\hline Sicily & 37.8501 & 14.4784 & 1089 & 806 \\
\hline Sicily & 37.8121 & 14.5096 & 980 & 648 \\
\hline Sicily & 37.7065 & 14.5348 & 837 & 613 \\
\hline Sicily & 37.6304 & 14.9407 & 700 & 613 \\
\hline Sicily & 37.6218 & 14.7419 & 733 & 407 \\
\hline Sicily & 37.6675 & 14.8363 & 570 & 497 \\
\hline Sicily & 37.5693 & 14.9106 & 240 & 447 \\
\hline Sicily & 37.5145 & 14.9681 & 254 & 494 \\
\hline Sicily & 37.4565 & 14.9127 & 26 & 435 \\
\hline Sicily & 37.6400 & 14.3927 & 640 & 622 \\
\hline Sicily & 37.4960 & 14.3880 & 588 & 598 \\
\hline Sicily & 37.5724 & 14.6931 & 185 & 440 \\
\hline Sicily & 37.4956 & 14.6451 & 500 & 545 \\
\hline Sicily & 37.4173 & 14.4468 & 816 & 737 \\
\hline Sicily & 37.3275 & 14.4473 & 469 & 587 \\
\hline Sicily & 37.2414 & 14.5186 & 513 & 514 \\
\hline Sicily & 37.2662 & 14.6909 & 524 & 601 \\
\hline Sicily & 37.3864 & 14.6960 & 257 & 452 \\
\hline
\end{tabular}




\begin{tabular}{lllrrrr} 
S3090 & Nicolosi & Sicily & 37.6153 & 15.0273 & 695 & 1123 \\
S3100 & Fleri & Sicily & 37.6568 & 15.0994 & 620 & 1158 \\
S3110 & Zafferana Etnea & Sicily & 37.6941 & 15.1063 & 590 & 1330 \\
S3120 & Linguaglossa & Sicily & 37.8411 & 15.1451 & 530 & 1156 \\
S3130 & Viagrande & Sicily & 37.6113 & 15.0991 & 405 & 954 \\
S3140 & Piedimonte Etneo & Sicily & 37.8083 & 15.1790 & 348 & 999 \\
S3150 & Acireale & Sicily & 37.6222 & 15.1659 & 194 & 817 \\
S3155 & Catania (Osservatorio) & Sicily & 37.5155 & 15.0872 & 75 & 609 \\
S3210 & Floresta & Sicily & 37.9878 & 14.9059 & 1270 & 1113 \\
S3270 & Roccella Valdemone & Sicily & 37.9357 & 15.0077 & 786 & 913 \\
S3280 & Francavilla di Sicilia & Sicily & 37.9058 & 15.1374 & 319 & 985 \\
S3310 & Taormina & Sicily & 37.8553 & 15.2886 & 248 & 711 \\
S3320 & Antillo & Sicily & 37.9780 & 15.2453 & 480 & 1263 \\
S3340 & Casalvecchio Siculo & Sicily & 37.9596 & 15.3231 & 420 & 889 \\
S3350 & Camaro & Sicily & 38.2111 & 15.5003 & 568 & 1041 \\
S3360 & Santo Stefano di Briga & Sicily & 38.1007 & 15.4813 & 139 & 875 \\
S3370 & Messina (Osservatorio) & Sicily & 38.2027 & 15.5541 & 54 & 803 \\
S3380 & Messina (Istituto Geofisico) & Sicily & 38.2022 & 15.5548 & 54 & 727 \\
S3400 & Ganzirri & Sicily & 38.2576 & 15.6090 & 1 & 705 \\
\hline
\end{tabular}


Table S2: Temperature stations used in the study and relative mean annual temperature in the period 1971-2000.

\begin{tabular}{|c|c|c|c|c|c|c|}
\hline Station ID & Station Name & Region & Latitude & Longitude & Elevation & Annual mean $\left({ }^{\circ} \mathrm{C}\right)$ \\
\hline C140 & Tricarico & Basilicata & 40.6173 & 16.1486 & 698 & 13.3 \\
\hline $\mathrm{C} 200$ & Matera & Basilicata & 40.6597 & 16.5954 & 450 & 15.2 \\
\hline $\mathrm{C} 230$ & Potenza & Basilicata & 40.6370 & 15.8021 & 811 & 12.4 \\
\hline $\mathrm{C} 500$ & Grumento Nova & Basilicata & 40.2909 & 15.8969 & 585 & 13.2 \\
\hline C620 & Stigliano & Basilicata & 40.3976 & 16.2274 & 908 & 12.3 \\
\hline $\mathrm{C} 810$ & Valsinni & Basilicata & 40.1673 & 16.4311 & 250 & 16.0 \\
\hline $\mathrm{C} 840$ & Nova Siri Scalo & Basilicata & 40.1340 & 16.6304 & 2 & 16.9 \\
\hline C930 & Villapiana Scalo & Calabria & 39.7973 & 16.4819 & 5 & 16.6 \\
\hline $\mathrm{C} 1010$ & Cosenza & Calabria & 39.2869 & 16.2653 & 242 & 16.7 \\
\hline C1100 & Cecita & Calabria & 39.4000 & 16.5378 & 1180 & 9.2 \\
\hline C1130 & Torano Scalo & Calabria & 39.4938 & 16.2098 & 97 & 16.3 \\
\hline $\mathrm{C} 1180$ & Castrovillari & Calabria & 39.7655 & 16.2542 & 353 & 15.0 \\
\hline $\mathrm{C} 1250$ & Fagnano Castello & Calabria & 39.5640 & 16.0556 & 516 & 15.0 \\
\hline $\mathrm{C} 1260$ & San Marco Argentano & Calabria & 39.5594 & 16.1108 & 430 & 15.3 \\
\hline C1330 & Rossano & Calabria & 39.5745 & 16.6343 & 300 & 16.1 \\
\hline C1460 & Ciro' Marina & Calabria & 39.4079 & 17.1093 & 6 & 17.0 \\
\hline $\mathrm{C} 1550$ & Trepido' & Calabria & 39.1887 & 16.6979 & 1295 & 9.5 \\
\hline $\mathrm{C} 1670$ & Cutro & Calabria & 39.0366 & 17.0152 & 169 & 16.9 \\
\hline $\mathrm{C} 1760$ & Botricello & Calabria & 38.9415 & 16.8586 & 18 & 17.4 \\
\hline $\mathrm{C} 1850$ & Catanzaro & Calabria & 38.9108 & 16.5859 & 334 & 16.2 \\
\hline $\mathrm{C} 1855$ & Santa Maria di Catanzaro & Calabria & 38.8496 & 16.5977 & 70 & 17.8 \\
\hline C1960 & Chiaravalle Centrale & Calabria & 38.6713 & 16.4088 & 714 & 13.5 \\
\hline C1970 & Soverato Marina & Calabria & 38.6917 & 16.5444 & 29 & 17.9 \\
\hline C1980 & Serra San Bruno & Calabria & 38.5652 & 16.3197 & 790 & 11.0 \\
\hline $\mathrm{C} 2120$ & Caulonia & Calabria & 38.3832 & 16.4114 & 275 & 16.7 \\
\hline $\mathrm{C} 2170$ & Siderno Marina & Calabria & 38.2741 & 16.2985 & 7 & 18.1 \\
\hline $\mathrm{C} 2180$ & Antonimina - Canolo Nuovo & Calabria & 38.3131 & 16.1272 & 880 & 12.4 \\
\hline $\mathrm{C} 2210$ & Ardore Superiore & Calabria & 38.2059 & 16.1550 & 250 & 17.9 \\
\hline $\mathrm{C} 2370$ & Melito di Porto Salvo & Calabria & 37.9200 & 15.7714 & 7 & 18.0 \\
\hline $\mathrm{C} 2450$ & Reggio Calabria & Calabria & 38.1078 & 15.6509 & 15 & 18.4 \\
\hline $\mathrm{C} 2470$ & Gambarie d'Aspromonte & Calabria & 38.1929 & 15.8449 & 1200 & 9.9 \\
\hline $\mathrm{C} 2600$ & Cittanova & Calabria & 38.3522 & 16.0775 & 407 & 16.1 \\
\hline $\mathrm{C} 2730$ & Mileto & Calabria & 38.6272 & 16.0669 & 368 & 15.7 \\
\hline $\mathrm{C} 2740$ & Rosarno & Calabria & 38.5005 & 15.9880 & 61 & 17.1 \\
\hline $\mathrm{C} 2770$ & Tropea & Calabria & 38.6753 & 15.8944 & 51 & 18.0 \\
\hline $\mathrm{C} 2890$ & Tiriolo & Calabria & 38.9395 & 16.5099 & 690 & 14.2 \\
\hline $\mathrm{C} 2960$ & Sant'Eufemia Lamezia & Calabria & 38.9215 & 16.2523 & 25 & 17.0 \\
\hline $\mathrm{C} 3000$ & Rogliano & Calabria & 39.1835 & 16.3257 & 650 & 14.1 \\
\hline
\end{tabular}




\begin{tabular}{|c|c|c|c|c|c|c|}
\hline C3050 & Fiumefreddo Bruzio & Calabria & 39.2351 & 16.0710 & 220 & 16.2 \\
\hline C3210 & Lagonegro & Basilicata & 40.1342 & 15.7620 & 666 & 12.7 \\
\hline $\mathrm{C} 3260$ & Maratea & Basilicata & 39.9840 & 15.7167 & 300 & 14.7 \\
\hline S140 & Tindari & Sicily & 38.1444 & 15.0449 & 280 & 17.5 \\
\hline S310 & San Fratello & Sicily & 38.0179 & 14.5990 & 690 & 16.3 \\
\hline S470 & Cefalù & Sicily & 38.0356 & 14.0170 & 30 & 19.3 \\
\hline S660 & Ciminna & Sicily & 37.8957 & 13.5598 & 525 & 16.3 \\
\hline $\mathrm{S} 720$ & Ficuzza & Sicily & 37.8868 & 13.3793 & 681 & 15.6 \\
\hline $\mathrm{S} 850$ & Partinico & Sicily & 38.0469 & 13.1177 & 189 & 18.2 \\
\hline S920 & Palermo (Piazza Verdi) & Sicily & 38.1210 & 13.3579 & 19 & 19.3 \\
\hline S950 & San Giuseppe Jato & Sicily & 37.9748 & 13.1869 & 462 & 18.3 \\
\hline S1030 & Trapani & Sicily & 38.0164 & 12.5072 & 2 & 18.6 \\
\hline S1050 & San Vito lo Capo & Sicily & 38.1901 & 12.7332 & 3 & 19.2 \\
\hline S1120 & Marsala & Sicily & 37.7990 & 12.4387 & 12 & 17.6 \\
\hline S1140 & Mazara del Vallo & Sicily & 37.6586 & 12.5994 & 8 & 18.7 \\
\hline S1180 & Partanna & Sicily & 37.7311 & 12.8922 & 407 & 17.4 \\
\hline S1270 & Corleone & Sicily & 37.8162 & 13.3019 & 588 & 16.7 \\
\hline S1380 & Sciacca & Sicily & 37.5130 & 13.0821 & 56 & 18.2 \\
\hline S1420 & Piano del Leone & Sicily & 37.6735 & 13.4668 & 831 & 14.1 \\
\hline S1480 & Bivona & Sicily & 37.6209 & 13.4396 & 521 & 17.3 \\
\hline S1490 & Lercara Friddi & Sicily & 37.7490 & 13.6005 & 658 & 15.7 \\
\hline S1700 & Racalmuto & Sicily & 37.4100 & 13.7341 & 450 & 17.7 \\
\hline S1810 & Agrigento (Ispettorato) & Sicily & 37.3130 & 13.5873 & 175 & 18.2 \\
\hline S1860 & Petralia Sottana & Sicily & 37.8092 & 14.0924 & 930 & 14.5 \\
\hline S1960 & Enna & Sicily & 37.5696 & 14.2731 & 950 & 13.9 \\
\hline S2020 & Caltanissetta & Sicily & 37.4978 & 14.0577 & 375 & 17.5 \\
\hline S2200 & Licata & Sicily & 37.1025 & 13.9343 & 70 & 18.6 \\
\hline S2240 & Gela & Sicily & 37.0652 & 14.2520 & 30 & 19.4 \\
\hline $\mathrm{S} 2250$ & Piazza Armerina & Sicily & 37.3673 & 14.3346 & 721 & 15.7 \\
\hline S2290 & Monterosso Almo & Sicily & 37.0890 & 14.7631 & 667 & 16.0 \\
\hline $\mathrm{S} 2350$ & Vittoria & Sicily & 36.9531 & 14.5266 & 168 & 17.4 \\
\hline S2370 & Ragusa & Sicily & 36.9242 & 14.7244 & 515 & 17.6 \\
\hline S2420 & Cozzo Spadaro & Sicily & 36.6884 & 15.1321 & 50 & 18.6 \\
\hline S2540 & Siracusa & Sicily & 37.0626 & 15.2928 & 23 & 18.5 \\
\hline S2580 & Lentini (Città) & Sicily & 37.2926 & 14.9989 & 43 & 19.0 \\
\hline S2690 & Cesarò & Sicily & 37.8469 & 14.7099 & 1100 & 13.0 \\
\hline S2900 & Adrano & Sicily & 37.6675 & 14.8363 & 570 & 17.2 \\
\hline S3050 & Caltagirone & Sicily & 37.2414 & 14.5186 & 513 & 16.7 \\
\hline S3090 & Nicolosi & Sicily & 37.6153 & 15.0273 & 695 & 15.8 \\
\hline S3110 & Zafferana Etnea & Sicily & 37.6941 & 15.1063 & 590 & 16.1 \\
\hline
\end{tabular}




\begin{tabular}{|c|c|c|c|c|c|c|}
\hline S3120 & Linguaglossa & Sicily & 37.8411 & 15.1451 & 530 & 15.6 \\
\hline S3150 & Acireale & Sicily & 37.6222 & 15.1659 & 194 & 18.3 \\
\hline S3155 & Catania (Osservatorio) & Sicily & 37.5155 & 15.0872 & 75 & 19.7 \\
\hline S3210 & Floresta & Sicily & 37.9878 & 14.9059 & 1270 & 11.5 \\
\hline S3310 & Taormina & Sicily & 37.8553 & 15.2886 & 248 & 20.6 \\
\hline S3380 & Messina (Istituto Geofisico) & Sicily & 38.2022 & 15.5548 & 54 & 18.6 \\
\hline
\end{tabular}

